\title{
Tecnura
}

http://revistas.udistrital.edu.co/ojs/index.php/Tecnura/issue/view/640

DOI: http://dx.doi.org/10.14483/udistrital.jour.tecnura.2014.SE1.a09

INVESTIGACIÓN

\section{Refrigeración de gabinetes de transmisión de datos por tubos de calor}

\author{
Refrigeration of data transmission boxes by heat pipe systems
}

Jorge Andrés Gaitán Triana*, Camilo Andrés Arias Henao**

Citation / Para citar este artículo: Gaitán Triana, J. A., \& Arias Henao, C. A. (2014). Refrigeración de gabinetes de transmisión de datos por tubos de calor. Revista Tecnura, Edición especial, 115-124.

Fecha de recepción: 29 de noviembre de 2013 / Fecha de aceptación: 11 de julio de 2014

\section{RESUMEN}

El proyecto de refrigeración de gabinetes de telecomunicaciones por medio de tubos de calor propone el diseño matemático y posterior simulación de un sistema de refrigeración por tubos de calor para disipar la energía generada por equipos de telecomunicaciones presentes en los gabinetes, evaluando la posible aplicación del modelo matemático en diferentes ambientes de Colombia. Este sistema de refrigeración permite disipar grandes cantidades de energía térmica en comparación con su sección de paso; además, no se requiere conectar los tubos a la red eléctrica (en algunos ambientes), lo que genera independencia de esta última.

Inicialmente se determina el modelo matemático que describe el funcionamiento del tubo, dependiendo de las necesidades de refrigeración. Este modelo es una ecuación de transferencia de calor que describe el paso de energía en las diferentes partes del tubo. Luego, en la simulación se presenta el comportamiento del sistema para un año típico, en el cual se obtienen datos de temperatura en el interior del gabinete, energía retirada por el banco de tubos, reducción del consumo de electricidad, entre otros datos. La simulación se aplica a diferentes ambientes de Colombia para evaluar el potencial del sistema en las regiones ecuatoriales.

Los resultados obtenidos en el proyecto tras la simulación en Trnsys 17 señalan la obtención de una temperatura relativamente estable en el funcionamiento de los equipos de telecomunicaciones refrigerados con los tubos de calor en la ciudad de Bogotá. En la simulación para ambientes más cálidos, como son las ciudades de Barranquilla y Cali, se logró una significativa reducción en la temperatura interior del gabinete, que es de aproximadamente $50 \%$.

Palabras clave: Cambio de fase, capilaridad, estado transitorio, flujo de calor, resistencia térmica.

\begin{abstract}
The cooling telecom cabinets project by heat pipes proposes the mathematical design and subsequent simulation of the refrigeration system by heat pipes for the dissipation of the energy generated by the telecommunications equipment in cabinets, evaluating the possible application of this mathematical model in different environments of Colombia. This cooling system allows dissipating large amounts of thermal energy compared to its flow section, moreover; it is not necessary to connect the pipes to the
\end{abstract}

\footnotetext{
* Ingeniero mecánico, estudiante de último semestre de pregrado en ingeniería mecánica, Universidad Distrital Francisco José de Caldas, Bogotá, Colombia. Contacto: jagaitant@correo.udistrital.edu.co

** Ingeniero mecánico. Magíster en ingeniería mecánica, candidato a doctor en ingeniería energética; docente, Universidad Distrital Francisco José de Caldas. Bogotá, Colombia. Contacto: carias@udistrital.edu.co
} 
grid (in some environments), generating independence from the latter.

Initially it is determined the mathematical model that describes the operation of the tube depending on the cooling requirements. This model is a heat transfer equation which describes the energy flow in the different parts of the tube. Then, the simulation shows the performance of the system for a typical year, in which it is obtained data related to the temperature inside the cabinet, removed energy by the tube bank, reduction in electricity consumption, among other data. The simulation is applied to different

\section{INTRODUCCIÓN}

Los sistemas electrónicos y de transmisión de datos requieren una adecuada temperatura para lograr un desempeño óptimo y una larga vida; para esto, se implementan sistemas de refrigeración que utilizan electricidad como fuente de energía, Ilevando a un aumento en el gasto económico.

La refrigeración por tubos de calor es un sistema que logra conducir grandes cantidades de calor a través de una sección de paso pequeña, ocupando poco espacio y, dependiendo de las condiciones climáticas y de funcionamiento, pueden independizarse de la red eléctrica, debido a que su flujo de energía es producido por el diferencial de temperaturas entre el ambiente y la zona a refrigerar.

Estos sistemas de refrigeración logran aportar soluciones a las nuevas políticas ambientales en el mundo ya que reducen los consumos de electricidad y su fluido de trabajo puede ser agua, alcoholes y fluidos amigables con el medio ambiente, constituyéndose así en una opción para los problemas energéticos y de contaminación.

El objeto de estudio se centra en determinar el potencial de los tubos de calor para refrigeración en gabinetes de transmisión de datos. Para esto se definirán los sistemas termodinámicos involucrados especificando los flujos de energía y environments in Colombia in order to evaluate the potential of the system in the Equatorial regions.

The results of the simulation in Trnsys 17 point out the obtaining of a relatively stable temperature in the operation of telecommunications equipment cooled with heat pipe in Bogotá city. In the simulation for warmer environments, such as Barranquilla and Cali, it was achieved a significant reduction in the temperature inside the cabinet that is approximately $50 \%$.

Keywords: Capillarity, heat flux, phase shift, thermal resistance, transient.

el desplazamiento del fluido en sus diferentes fases para seleccionar así los materiales a utilizar en el modelo matemático y simular el comportamiento del gabinete y los tubos, obteniendo al final el potencial teórico del sistema de tubos de calor.

En la simulación del gabinete en las tres diferentes ciudades se encontró que se hace una reducción significativa de las temperaturas, estando en un promedio de $50 \%$ con respecto a las temperaturas máximas que superan $100{ }^{\circ} \mathrm{C}$, por la generación externa y las ganancias por condiciones ambientales. Al final se concluye que siendo un sistema de refrigeración pasivo que no consume energía de fuentes externas, se logra reducir la temperatura externa en un porcentaje considerable.

\section{DESARROLLO}

\section{Diseño analítico del tubo de calor}

En el diseño del tubo de calor, lo primero que se debe hacer es definir el sistema térmico (Jones \& Dugan, 1997) con las cargas que se desea refrigerar, los flujos energéticos, el ciclo de vapor generado para la refrigeración y el diferencial de temperaturas entre el ambiente y el lugar que se va a refrigerar. 
En la figura 1 se aprecia un esquema del sistema térmico, en donde se definen los límites, el desplazamiento del fluido en estados líquido y gaseoso; los flujos energéticos que se representan en los extremos del tubo (National Aeronautics and Space Administration, 1979).

En los gabinetes de transmisión de datos, se extraerá energía del aire presente en el sistema por medio del evaporador del tubo de calor, y el condensador estará al aire libre; con esto se conseguirá refrigerar el gabinete, evitando el consumo de electricidad en otro tipo de sistemas de refrigeración; además, al no poseer elementos móviles se evita la adición de ruido al sistema, ya que para estas aplicaciones se debe tener un máximo de $\mathrm{dB}$ en el día de 55 y 50 en la noche.

\section{Determinación del tubo}

Para determinar el tubo de calor más indicado para la aplicación en los gabinetes de transmisión de datos debemos especificar: las temperaturas de operación del sistema, la carga energética que se desea disipar, el espacio, la geometría y la orientación del banco de tubos.

Se tomará un rango de temperatura de operación de 10 a $60{ }^{\circ} \mathrm{C}$ (son las temperaturas extremas a las que se verá sometido el fluido de trabajo) para el diseño de los tubos, y la temperatura dentro del gabinete será de aprox. $30{ }^{\circ} \mathrm{C}$ para tener un

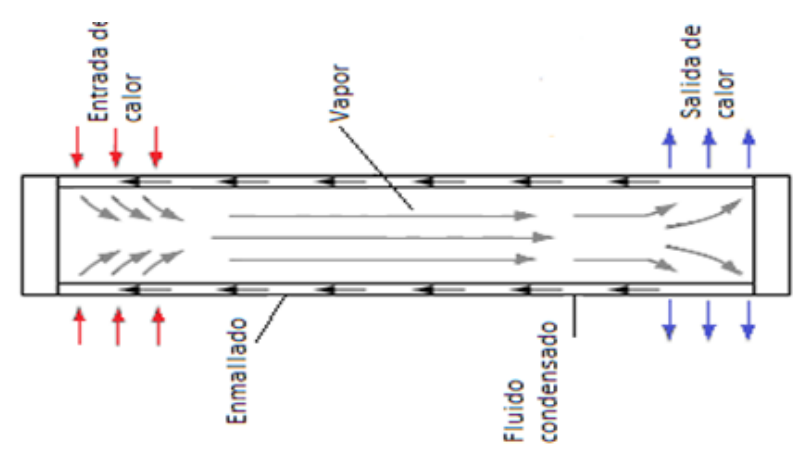

Figura 1. Esquema térmico.

Fuente: Elaboración propia. óptimo desempeño de los equipos de telecomunicaciones; el flujo de calor que se desea retirar es de 5000 Btu/h (aprox. 1465 W).

Después de definir las condiciones de funcionamiento del sistema de tubos de calor, se designará el cobre como material para la construcción de los tubos, ya que este metal tiene una conductividad térmica alta y es compatible con varios fluidos de trabajo.

El espacio para la implementación de los tubos de calor se restringe a $5 \mathrm{~cm}$ de espesor, lo cual determina el diámetro máximo de los tubos. Se seleccionarán diámetros comerciales para facilitar una posible construcción (ver la figura 2).

Se seleccionó tubería de cobre de $1 / 4$ in (para la parte interna), debido a su fácil adquisición y manejo, además por sus dimensiones se adapta al espacio en el gabinete en donde se instalará. Esta tubería nos da un área de sección de vapor de $1,642 \times 10^{-5} \mathrm{~m}^{2}(\mathrm{r}=2,286 \mathrm{~mm})$. La tubería externa será de $3 / 8$ de pulgada.

\section{Fluido de trabajo}

Para determinar los posibles fluidos de trabajo se debe considerar que estos sean compatibles con el material del tubo, ya que se podrían presentar problemas de oxidación, generación de burbujas por reacciones químicas y degradación del tubo. A continuación se indican algunos fluidos

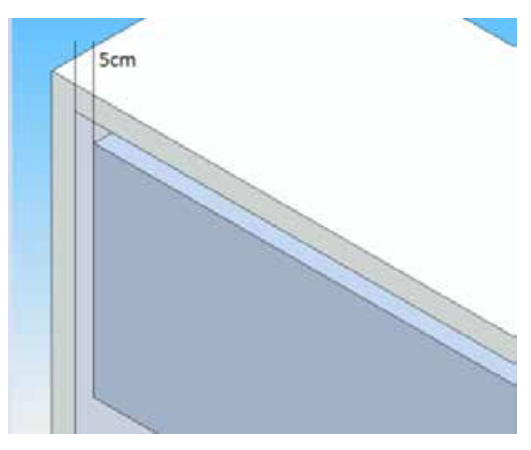

Figura 2. Esquema del espacio en donde se instalarán los tubos.

Fuente: Elaboración propia. 
compatibles con el cobre: acetona, amoniaco, agua, Dow-A y Dow-E. Se seleccionará el agua debido a su mayor conductividad térmica.

En los tubos de calor encontramos diferentes tipos de limitaciones, las cuales nos delimitan la cantidad de energía que puede retirar el sistema. En la figura 3 se aprecian las curvas de límites.

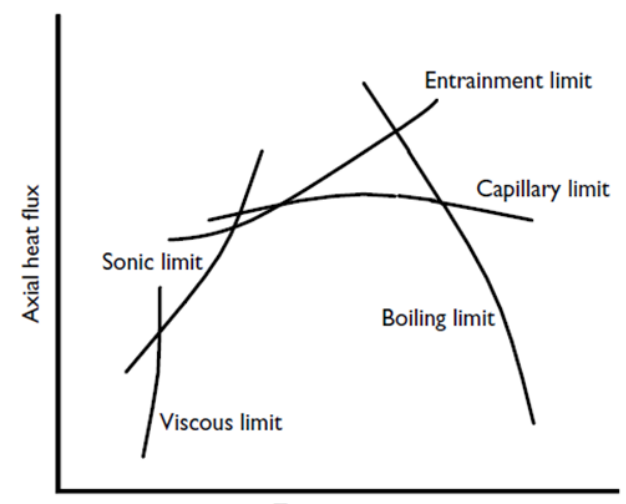

Temperature

Figura 3. Límites en el flujo de calor.

Fuente: Reay, Kew, \& McGlen (2006).

Para la temperatura más baja $\left(\mathrm{T}_{1}=10{ }^{\circ} \mathrm{C}\right)$ la limitación sónica determina el menor flujo de calor axial (Reay, Kew, \& McGlen, 2006), el cual viene representado por la ecuación (1):

$$
\dot{\mathrm{q}}_{\mathrm{s}}=\rho_{\mathrm{v}} \mathrm{h}_{\mathrm{fg}} \sqrt{\frac{\gamma \mathrm{RT}_{\mathrm{v}}}{2(\gamma+1)}}
$$

Donde (Miranda Barreras, 2005):

- $\dot{q}_{s}=$ Límite sónico $\left(\mathrm{W} / \mathrm{m}^{2}\right)$

- $\rho_{v}=$ Densidad del vapor $\left(\mathrm{kg} / \mathrm{m}^{3}\right)$

- $R=$ Constante del gas $(\mathrm{kJ} / \mathrm{kgK})$

- $T_{v}=$ Temperatura de vapor $(\mathrm{K})$

- $\gamma=$ Cociente de los calores específicos

- $h_{f g}=$ Calor latente $(\mathrm{kJ} / \mathrm{kg})$

El valor de $\mathrm{R}$ es $0,462 \mathrm{~kJ} / \mathrm{kgK}$
Se calcula el límite sónico con las propiedades del agua:

$$
\dot{q}_{s}=3,767 \times 10^{6} \mathrm{~W} / \mathrm{m}^{2}
$$

Determinado el potencial del tubo en la temperatura más baja de operación, ahora se calcula el máximo flujo de energía posible a la temperatura más alta $\left(T_{h}\right)$ para el amoniaco, que es determinado por el límite de arrastre. Para calcular este parámetro se debe hacer referencia al número de Weber (We), ecuación (2) (Miranda Barreras, 2005), el cual establece la relación entre las fuerzas de inercia y las de tensión superficial.

$$
W_{e}=\frac{\rho_{v} c_{v}^{2} L}{2 \pi \sigma}
$$

Donde: = densidad del vapor; = velocidad del vapor; = tensión superficial de la interface líquido-vapor; = longitud característica.

Para poder calcular el máximo flujo de calor, se considera que este ocurre cuando las fuerzas de inercia se igualan a las de tensión superficial, entonces $W_{e}=1$. De acuerdo con la anterior suposición, el límite se calcula con la ecuación (3) (Miranda Barreras, 2005):

$$
\dot{Q}_{e n t}=\pi r_{v}^{2} h_{f g} \sqrt{\frac{2 \pi \rho_{v} \sigma}{z}}
$$

Se hace referencia a z como la dimensión característica de la interface líquido-vapor y puede tomarse como 0,036 mm. El límite de arrastre se evalúa con la temperatura más alta.

$$
\mathrm{h}_{\mathrm{fg}}=\mathrm{J} / \mathrm{kg} ;=\mathrm{N} / \mathrm{m} ;=\mathrm{kg} / \mathrm{m}^{3} ; \mathrm{r}_{\mathrm{v}}=\mathrm{m} ; \mathrm{z}=\mathrm{m}
$$

Después del cálculo con la ecuación (3) obtenemos el siguiente resultado: 


$$
\dot{Q}_{\text {ent }}=1424,5 \mathrm{~W}
$$

El mayor flujo de calor del fluido a máxima temperatura es de 1424,5 W.

Se determina usar agua como fluido de trabajo, ya que de los posibles fluidos es el de mejor comportamiento.

\section{Enmallado del tubo de calor}

Para la selección del enmallado se escogerá esferas empacadas, que es un tipo de enmallado compuesto por esferas sinterizadas, el cual posee el mayor flujo de calor de los enmallados homogéneos, además logra vencer fuerzas gravitacionales.

Se utilizarán esferas sinterizadas de 0,458 mm de diámetro con una conductividad térmica de 31 $\mathrm{W} / \mathrm{mK}$ y una porosidad $\varepsilon=0,63$. (Patiño \& Figueroa, 2004).

La conductividad efectiva $\left(k_{e f}\right)$ representa la capacidad de transferencia de calor del conjunto reIleno-fluido. Para esto se utiliza la ecuación (4) (Miranda Barreras, 2005).

$$
\kappa_{e f}=\frac{\kappa_{1}\left[\left(2 \kappa_{1}+\kappa_{w}\right)-2(1-\varepsilon)\left(\kappa_{1}-\kappa_{w}\right)\right]}{\left[2 k_{1}+k_{w}+(1-\varepsilon)\left(\kappa_{1}-\kappa_{w}\right)\right]}
$$

Donde:

- = conductividad del fluido agua $(0,611 \mathrm{~W} / \mathrm{mK})$

- = conductividad del material del relleno aluminio $(31 \mathrm{~W} / \mathrm{mK})$

\section{Modelo matemático}

El tubo de calor es un dispositivo que transfiere energía de una temperatura más alta a una más baja, por este motivo se representa con una ecuación de transferencia de calor, en donde el coeficiente global de transferencia de calor se calcula con la sumatoria de las inversas de las resistencias térmicas de cada sección por donde circula el flujo energético. En la figura 4 se aprecia un esquema de las resistencias térmicas que representan al tubo y en la tabla 1 se describen las convenciones utilizadas.

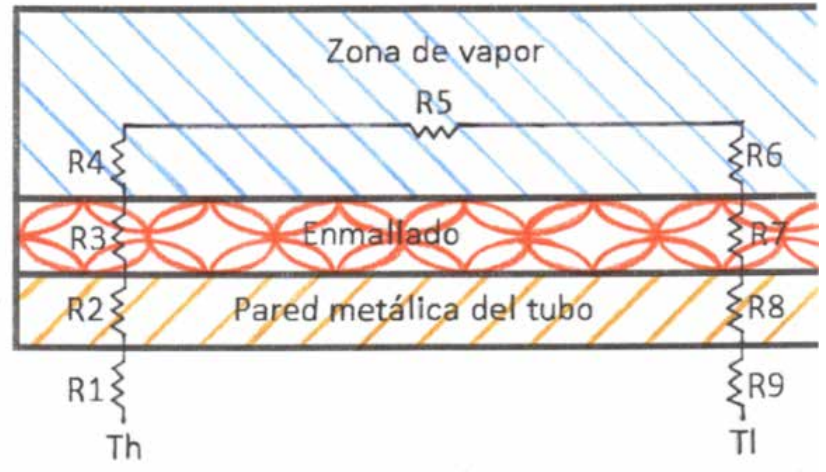

Figura 4. Esquema de resistencias térmicas.

Fuente: Elaboración propia.

Tabla 1. Representación de las resistencias a través del tubo.

\begin{tabular}{cl}
\hline Representación & \multicolumn{1}{c}{ Significado } \\
\hline R1 & $\begin{array}{l}\text { Resistencia por convección entre el aire } \\
\text { caliente y el tubo en el evaporador. }\end{array}$ \\
\hline R2 & $\begin{array}{l}\text { Conducción por la pared metálica por } \\
\text { el lado del evaporador. }\end{array}$ \\
\hline R3 & $\begin{array}{l}\text { Conducción por el relleno por el lado } \\
\text { del evaporador. }\end{array}$ \\
\hline R4 & $\begin{array}{l}\text { Convección entre relleno y vapor en el } \\
\text { evaporador. }\end{array}$ \\
\hline R5 & Flujo axial por el vapor. \\
\hline R6 & $\begin{array}{l}\text { Convección entre vapor y relleno en el } \\
\text { condensador. }\end{array}$ \\
\hline R7 & $\begin{array}{l}\text { Conducción por el relleno en el evapo- } \\
\text { rador. }\end{array}$ \\
\hline R8 & $\begin{array}{l}\text { Conducción por la pared del tubo en el } \\
\text { condensador. }\end{array}$ \\
\hline R9 & $\begin{array}{l}\text { Convección en el exterior entre la pared } \\
\text { y un flujo frío. }\end{array}$ \\
\hline
\end{tabular}

Fuente: Elaboración propia.

Para simplificar los cálculos las resistencias térmicas se multiplicarán por el área transversal para manejar resistencias específicas. Además se supondrá que las resistencias convectivas son muy pequeñas y se omitirán en los cálculos, de manera que entonces los cálculos se reducen a: 
- A través de la pared del evaporador

$$
r_{w e}=\frac{r_{0} \varepsilon_{w}}{2 k_{w} L_{e}}
$$

- A través del relleno del lado del evaporador

$$
r_{r e}=\frac{r_{0}^{2} \varepsilon_{r}}{2 k_{r} r_{i} L_{e}}
$$

- La resistencia específica axial

$$
r_{v}=\frac{\pi r_{0}^{2} F_{v} T_{v} L_{e f}}{\rho_{v} h_{f g}}
$$

- A través del relleno del lado del condensador

$$
r_{r c}=\frac{r_{0} \varepsilon_{r}}{2 k_{r} L_{c}}
$$

- A través de la pared del condensador

$$
r_{w c}=\frac{r_{0}^{2} \varepsilon_{w}}{2 k_{w} r_{i} L_{c}}
$$

- La resistencia total

$$
r_{T C}=\frac{1}{U_{T C}}=r_{w e}+r_{r e}+r_{v}+r_{r c}+r_{w c}
$$

Para el cálculo de las resistencias se debe tener en cuenta:

$r_{0}=$ radio exterior $0,0048 \mathrm{~m}$

$r_{i}=$ radio interior del tubo $0,0039 \mathrm{~m}$

$\varepsilon_{w}=$ grosor de la pared del tubo $9 \times 10^{-4} \mathrm{~m}$

$\varepsilon_{r}=$ grosor del relleno $0,00069 \mathrm{~m}$

$k_{w}=$ conductividad de la pared del tubo 401 $\mathrm{W} / \mathrm{mK}$

$L_{e}=$ longitud del evaporador 0,05 m

$L_{c}=$ longitud del condensador $0,2 \mathrm{~m}$

$L_{a}=$ longitud zona adiabática $1 \mathrm{~m}$

$L_{e f}=$ longitud efectiva $1,0417 \mathrm{~m}$

$F_{v}=$ coeficiente de fricción 13,6 s/m $\mathrm{m}^{4}$

$T_{v}=$ temperatura de vapor $300,15 \mathrm{~K}$

$\rho_{v}=$ densidad del vapor $0,025 \mathrm{~kg} / \mathrm{m}^{3}$

$h_{f g}=$ calor latente $2437 \mathrm{~kJ} / \mathrm{kg}$

Se calculan las resistencias específicas con los valores antes mencionados utilizando el programa
HEATPIPE, el cual utiliza la ecuación (5) para la conductividad a través de la pared del tubo, la ecuación (6) para la conductividad a través del relleno, la ecuación (7) es la generada axialmente por conducción y convección en el tubo; en la zona del condensador encontramos la ecuación (8) para el relleno al lado del condensador y la ecuación (9) para la pared del tubo; finalmente, la ecuación (10) es la sumatoria de todas las demás resistencias. Los resultados calculados se resumen a continuación:

$$
\begin{gathered}
r_{w e}=1,0773 \times 10^{-7} \mathrm{~m}^{2} \mathrm{~K} / \mathrm{W} \\
r_{r e}=9,347 \times 10^{-6} \mathrm{~m}^{2} \mathrm{~K} / \mathrm{W} \\
r_{v}=2,643 \times 10^{-6} \mathrm{~m}^{2} \mathrm{~K} / \mathrm{W} \\
r_{r c}=2,336 \times 10^{-6} \mathrm{~m}^{2} \mathrm{~K} / \mathrm{W} \\
r_{w c}=0,345^{-6} \mathrm{~m}^{2} \mathrm{~K} / \mathrm{W} \\
r_{T C}=1,6048 \times 10^{-5} \mathrm{~m}^{2} \mathrm{~K} / \mathrm{W} \\
r_{T C}=r_{w e}+r_{r e}+r_{v}+r_{r c}+r_{w c} \\
U_{T C}=\frac{1}{r_{T C}}=62313 \mathrm{~W} / \mathrm{m}^{2} \mathrm{~K}
\end{gathered}
$$

Mediante la ecuación (11) se calcula el área de transferencia de calor:

$$
A_{t}=\pi r_{0}^{2}
$$

Por último, la ecuación (12) (Incropera \& De Witt, 1999) es la ecuación de transferencia de calor con la cual se simula el tubo:

$$
\dot{Q}=A_{t} U_{T C}\left(T_{w e}-T_{w c}\right)
$$

$\mathrm{T}_{\text {we }}=$ temperatura de la pared en el evaporador

$\mathrm{T}_{\mathrm{wc}}=$ temperatura de la pared en el condensador

$$
\dot{Q}=\left(7,238 \times 10^{-5} \mathrm{~m}^{2}\right)(62313 \mathrm{~W} / \mathrm{m} 2 \mathrm{~K})\left(30^{\circ} \mathrm{C}-T_{w c}\right)
$$

Para determinar la máxima cantidad de energía que puede retirar el tubo de calor, se calculan las diferentes limitaciones; en la tabla 2 se resumen los resultados obtenidos en el programa HEATPIPE, el cual hace un cálculo iterativo. 
Tabla 2. Resumen de limitaciones.

\begin{tabular}{ll}
\hline \multicolumn{1}{c}{ Tipo de limitación } & \multicolumn{1}{c}{ Limitación $(\mathbf{W})$} \\
\hline Capilar & 531 \\
\hline Viscosa & 4831 \\
\hline Sónica & 248,43 \\
\hline Arrastre & 143,16 \\
\hline Ebullición & Mayor que 10000 \\
\hline
\end{tabular}

Fuente: Elaboración propia.

\section{METODOLOGÍA}

\section{Desarrollo del modelo computacional}

La simulación del sistema de tubos de calor se efectuará mediante el software TRNSYS, el cual permite hacer un análisis en estado transitorio, visualizando temperaturas y flujos de calor que son los datos representativos del sistema.
Para representar el gabinete en TRNSYS se utiliza la unidad 56 (University of Wisconsin-Madison, 2004), en la cual se introducen las dimensiones, se define el material de las paredes, las infiltraciones de aire, las ganancias energéticas y entradas para refrigeración. En la figura 6 se aprecia el montaje de las unidades.

Para introducir los tubos de calor, primero se programa la ecuación de transferencia de calor que representa el sistema en Microsoft Office Excel 2007, limitando el flujo máximo de calor 143,16 W por tubo. Después de esto se introduce al entorno de TRNSYS por medio de la unidad type 62 mostrada en la figura 5.

Se programa el archivo para que represente el flujo de calor de 6 tubos de las mismas características (insertando una nueva ecuación), dando como resultado la refrigeración del gabinete de transmisión de datos.

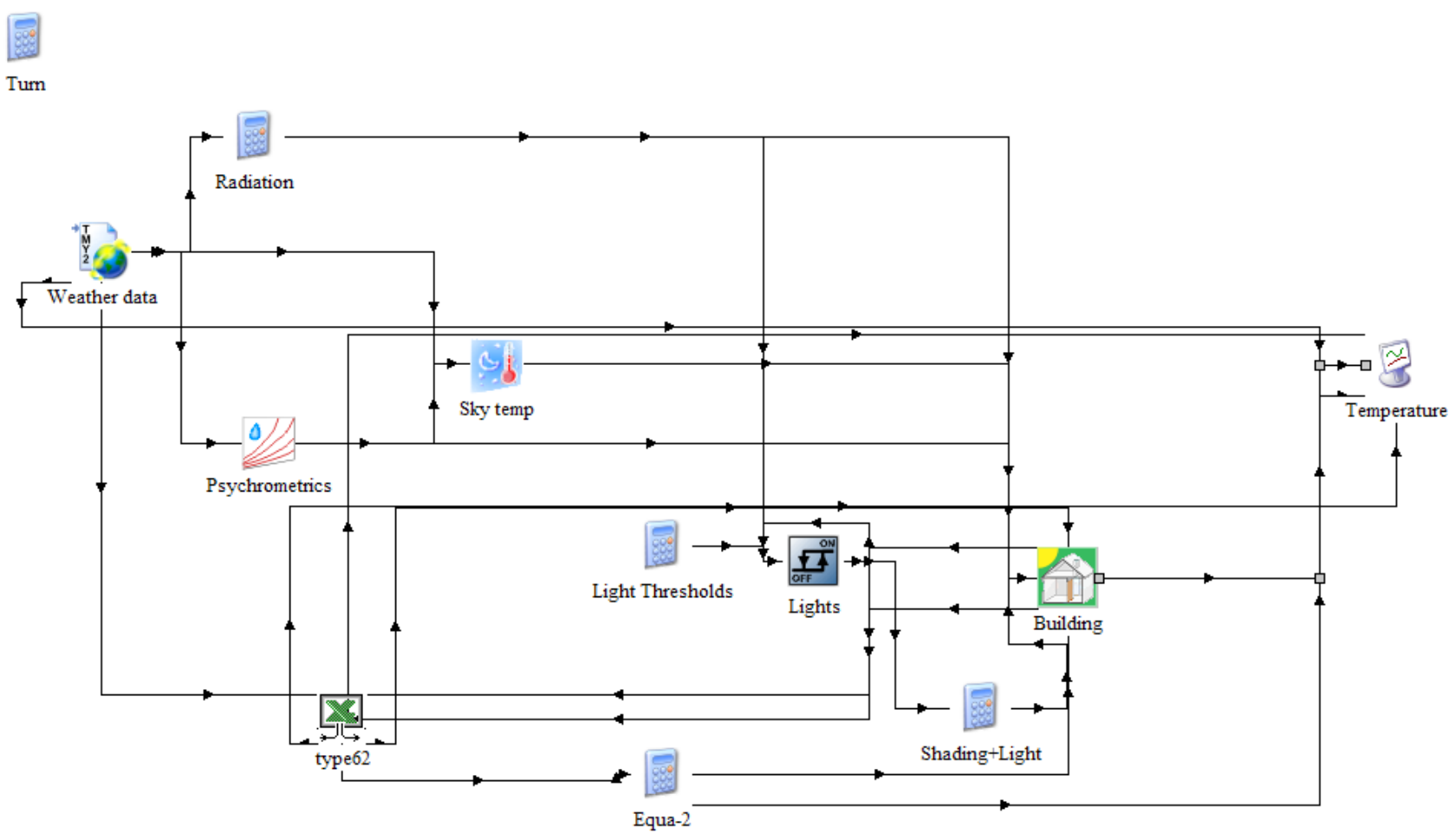

Figura 5. Montaje de las unidades en TRNSYS.

Fuente: University of Wisconsin-Madison (2004). 


\section{RESULTADOS}

El diseño del sistema de refrigeración por tubos de calor se evaluará en tres ciudades diferentes, Bogotá, Cali y Barranquilla, pues cada una de ellas presenta diferencias climáticas; en esta forma se podrá apreciar el potencial de los tubos de calor.

En la simulación del sistema con 6 tubos se logró disminuir la temperatura dentro del sistema a un promedio de $54{ }^{\circ} \mathrm{C}$ para la ciudad de Bogotá, como se aprecia en la figura 6 . En esta gráfica se observa que el comportamiento de la temperatura dentro del sistema es similar a la temperatura ambiente, esto se debe a que los tubos están disipando $143 \mathrm{~W}$ (constante), que es su límite, lo que da como resultado una refrigeración invariable. Aunque no se logra satisfacer la necesidad de mantener $30^{\circ} \mathrm{C}$ constantes, sí se logra una reducción de temperatura que oscila en $50 \%$ de la temperatura del gabinete sin equipos de refrigeración (promedio de $110^{\circ} \mathrm{C}$ sin equipos).

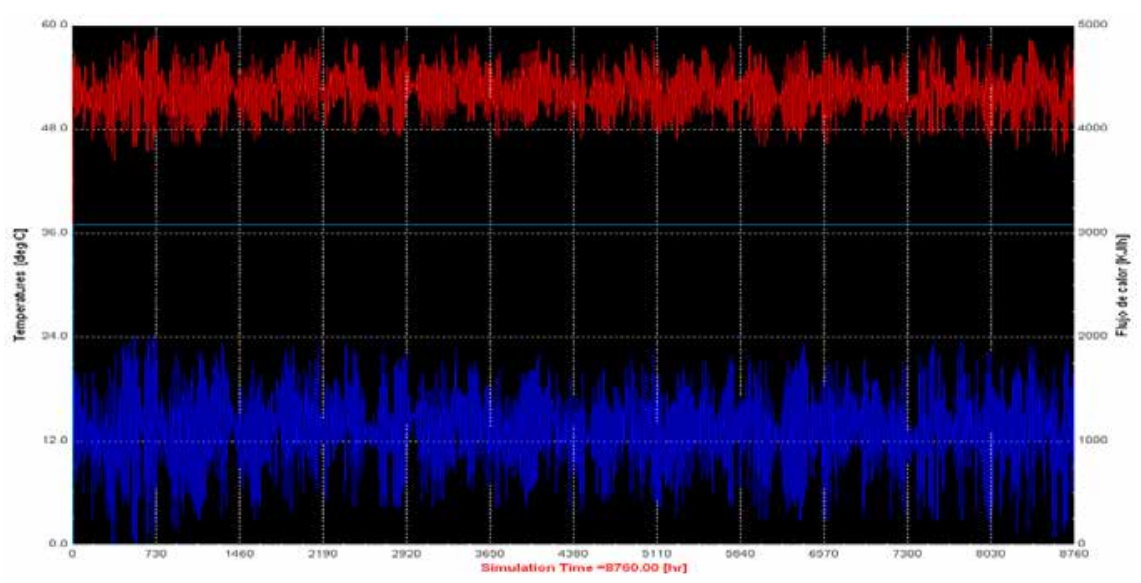

Figura 6. Bogotá. Temperatura ambiente (azul). Temperatura interior (rojo). Refrigeración (azul claro).

Fuente: University of Wisconsin-Madison (2004).

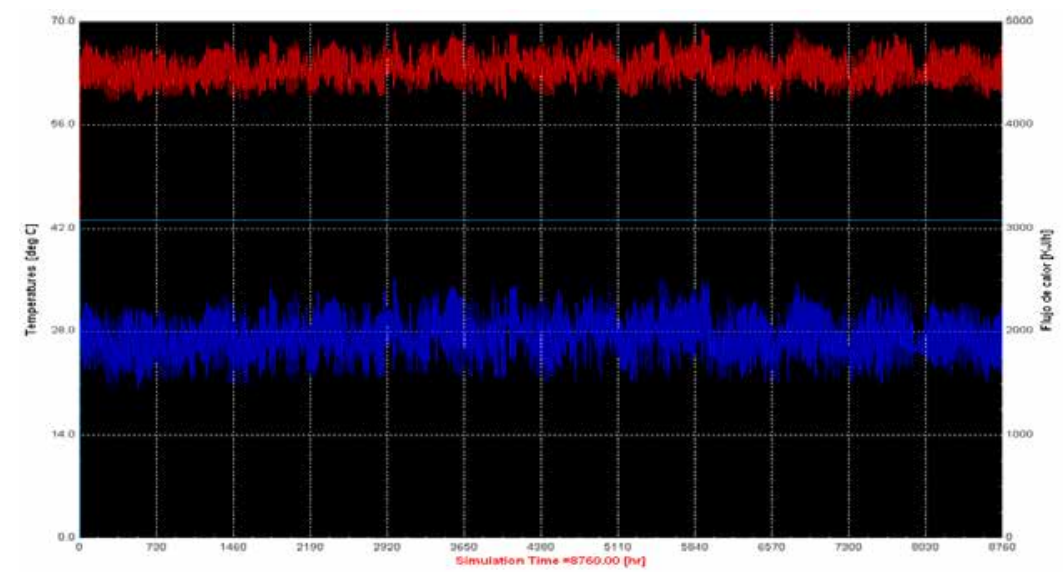

Figura 7. Barranquilla. Temperatura ambiente (azul). Temperatura interior (rojo). Refrigeración (azul claro).

Fuente: University of Wisconsin-Madison (2004). 


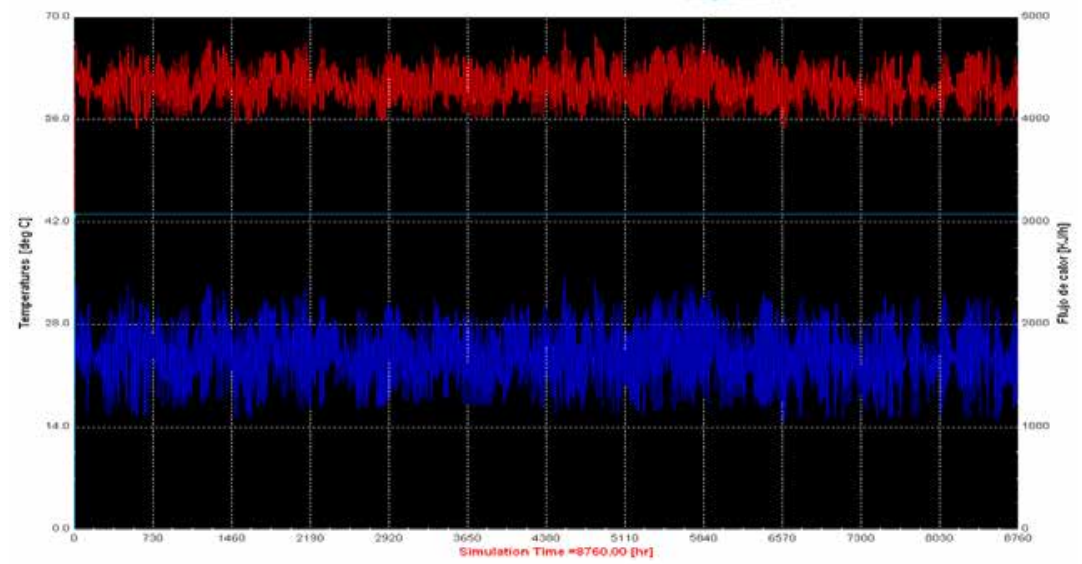

Figura 8. Cali. Temperatura ambiente (azul). Temperatura interior (rojo). Refrigeración (azul claro).

Fuente: University of Wisconsin-Madison (2004).

El comportamiento en las temperaturas de la ciudad de Bogotá es muy similar al que se presenta en las simulaciones hechas para Barranquilla (figura 7) y Cali (figura 8), con la diferencia de que las temperaturas interiores son en promedio 62 y $60{ }^{\circ} \mathrm{C}$, respectivamente.

La temperatura dentro del gabinete tiende a comportarse de modo muy parecido a la temperatura ambiente, pero con variaciones muy marcadas (figura 7, línea roja), lo cual se produce por la relación cíclica con la que efectúa el cálculo el programa Trnsys. Los flujos de energía son constantes porque el sistema se diseñó para que refrigerara todo el tiempo (8760 h), y así evitar inconvenientes de interpolaciones cíclicas.

\section{ANÁLISIS DE RESULTADOS}

Se logra una reducción significativa de la temperatura en el interior de los gabinetes, como apreciamos en las gráficas 7, 8 y 9. Aunque no se logran temperaturas óptimas $\left(30^{\circ} \mathrm{C}\right)$, se mejora las condiciones de operación sin necesidad de consumir energía de la red eléctrica.

La ecuación que representa los tubos de calor es dependiente de un diferencial de temperaturas (Ec. 12), por esta razón la temperatura dentro del sistema debe ser superior a la ambiente para poder disipar la carga requerida.

Para lograr mejores resultados en ciudades de climas diferentes a Bogotá, es necesario realizar diseños particulares debido a que manejan diferenciales de temperatura diferentes.

Al aumentar la longitud del evaporador y la del condensador y reducir la longitud de la zona adiabática se obtendrán mejores resultados, ya que al efectuar el cálculo de las resistencias térmicas la longitud del evaporador y del condensador son inversamente proporcionales a la resistencia, aumentando la conductividad térmica del tubo; por el contrario, la longitud de la zona adiabática es directamente proporcional a la resistencia especifica axial, lo que genera que la conductividad térmica disminuya.

El fluido de trabajo es uno de los parámetros más importantes del diseño, ya que es el que realiza el proceso; por esto se debe tener en cuenta que su temperatura de operación sea la correcta (Reay, Kew, \& McGlen, 2006); además, cuanto mayor sea la conductividad del fluido, mayor será la conductividad efectiva en el relleno del tubo.

\section{FINANCIAMIENTO}

Universidad Distrital Francisco José de Caldas 


\section{REFERENCIAS}

Incropera, F., \& De Witt, D. (1999). Fundamentos de Transferencia de Calor (Cuarta ed.). Mexico D.F., Mexico: Prentice Hall.

Jones, J., \& Dugan, R. (1997). Ingeniería Termodinámica. Mexico D.F:, Mexico: Prentice-Hall.

Miranda Barreras, Á. L. (2005). Tubos de calor. Una tecnología para el siglo XXI. Barcelona, España: Ediciones CEAC.

National Aeronautics and Space Administration. (1979). Heat Pipe Design Handbook. Towson, Maryland, Estados Unidos: B \& K Engineering.
Patiño, L., \& Figueroa, R. (2004). Convección de calor en el flujo de fluidos a través de un lecho de empaque de bauxita activada. Revista Facultad De Ingeniería, U.T.A., 12(2), 27-34.

Reay, D., Kew, P., \& McGlen, R. (2006). Heat Pipes Theory, Design and Applications (5 ed.). Middlesex, Massachusetts, Estados Unidos: Butterworth-Heinemann.

University of Wisconsin-Madison, S. (2004). TRNSYS 17, Multizone Building Modeling with Type 56 and TRNBuild (Vol. 6). Madison, Wisconsin, Estados Unidos: University of Wisconsin-Madison. 